\title{
Cost-effectiveness of ranibizumab in treatment of diabetic macular oedema (DME) causing visual impairment: evidence from the RESTORE trial
}

\author{
Paul Mitchell, ${ }^{1}$ Lieven Annemans, ${ }^{2}$ Meghan Gallagher, ${ }^{3}$ Rafiq Hasan, ${ }^{3}$ Simu Thomas, ${ }^{4}$ \\ Kerry Gairy, ${ }^{5}$ Martin Knudsen, ${ }^{6}$ Henrietta Onwordi ${ }^{6}$
}

- Additional materials are published online only. To view these files please visit the journal online (http://bjo.bmj. com/content/96/5.toc).

${ }^{1}$ Centre for Vision Research, Westmead Millennium Institute, University of Sydney, Sydney, NSW, Australia

${ }^{2}$ Ghent University, Ghent, Belgium

${ }^{3}$ Novartis Pharma AG, Basel, Switzerland

${ }^{4}$ Novartis Pharmaceuticals Corporation, East Hanover, New Jersey, USA

${ }^{5}$ Novartis Pharmaceuticals, Frimley, UK

${ }^{6}$ IMS Health, London, UK

\section{Correspondence to}

Dr Professor Paul Mitchell,

Centre for Vision Research, Westmead Millennium Institute, Westmead Hospital

Hawkesbury Rd, Westmead 2145 Australia;

paul.mitchell@sydney.edu.au

Accepted 2 November 2011

Published Online First

7 March 2012

\begin{abstract}
Background/aims To evaluate the cost-effectiveness of ranibizumab as either monotherapy or combined with laser therapy, compared with laser monotherapy, in the treatment of diabetic macular oedema (DME) causing visual impairment from a UK healthcare payer perspective.

Methods A Markov model simulated long-term outcomes and costs of treating DME in one eye (BCVA $\leq 75$ letters) based on data from the RESTORE Phase III trial. Outcomes measured in quality-adjusted life-years (OALYs) were simulated for a 15-year time horizon based on 12-month follow-up from RESTORE and published long-term data. Costs included treatment, disease monitoring, visual impairment and blindness (at 2010 price levels).

Results Ranibizumab monotherapy resulted in a 0.17 OALY gain at an incremental cost of $£ 4191$ relative to laser monotherapy, yielding an incremental costeffectiveness ratio (ICER) of $£ 24028$. Probabilistic sensitivity analysis showed a $64 \%$ probability of being cost-effective at a threshold of $£ 30000$ per QALY. Combined ranibizumab and laser therapy resulted in a 0.13 QALY gain at an incremental cost of $£ 4695$ relative to laser monotherapy (ICER $£ 36106$; $42 \%$ probability of ICER $<£ 30000$ ).
\end{abstract}

Conclusions Based on RESTORE 1-year follow-up data, ranibizumab monotherapy appears to be cost-effective relative to laser monotherapy, the current standard of care. Cost-effectiveness of combination therapy is less certain. Ongoing studies will further inform on disease progression and the need for additional ranibizumab treatment.

\section{INTRODUCTION}

Diabetic macular oedema (DME) is the most frequent cause of vision impairment in people with diabetes and can lead to blindness if left untreated. Even when patients receive optimal treatment with the current standard of care for DME, laser photocoagulation, improvements in vision are relatively uncommon and many patients lose vision despite laser therapy. ${ }^{12}$ This continued vision loss is the result of structural and physiological damage to the retinal capillary bed, and from progressive and permanent damage to the macular pigment epithelium, associated with poor control of blood glucose, blood pressure and lipid levels (the three main systemic risk factors for diabetic retinopathy and DME). ${ }^{3}$

The UK prevalence of visual impairment due to DME is estimated at approximately $3 \%$ of the adult (aged $18+$ years) diabetic population. ${ }^{4}$ Visual impairment places a substantial socio-economic burden on patients, their caregivers and healthcare systems at large. ${ }^{5}$ There is, therefore, a strong public health incentive to choose safe therapies that provide increased health gains through improved vision and patient functioning, while offering an acceptable balance between benefits and costs.

Ranibizumab (Lucentis ${ }^{\circledR}$, Novartis Pharma AG, Switzerland) is a novel agent that is currently licensed for the treatment of visual impairment due to DME. ${ }^{6}$ Ranibizumab selectively inhibits active isoforms of human vascular endothelial growth factor A (VEGF-A) from binding to its receptors. VEGF-A stimulates growth of new blood vessels and is a major mediator of increased vascular leakage,$^{7-10}$ mechanisms thought to be associated with retinal damage and progression of DME and the resulting visual impairment.

The Phase III RESTORE trial enrolled 345 patients with visual impairment due to DME and assessed ranibizumab given either as monotherapy or in combination with laser photocoagulation, compared with laser photocoagulation alone. The results showed that ranibizumab alone or in combination with laser provided significantly greater improvements in best corrected visual acuity (BCVA) at 1 year compared with laser therapy alone, with mean average BCVA changes of +6.1 and +5.9 versus +0.8 letters, respectively. ${ }^{11}$ In addition, health-related quality of life, as assessed using the National Eye Institute Visual Function Questionnaire (NEI VFQ-25), was improved significantly from baseline for both ranibizumab treatment groups compared with laser therapy alone $(\mathrm{p}<0.05$ for composite score and visionrelated subscales). RESTORE is ongoing in a 2-year open-label extension phase.

This report summarises the results of a health economic model that was developed based on RESTORE trial data to evaluate the cost-effectiveness of ranibizumab from a UK payer perspective when used as monotherapy or in combination with laser therapy compared with laser therapy alone. The model was developed separately from current assessments of ranibizumab by the UK Health Technology Assessment bodies, such as the National Institute for Health and Clinical Excellence.

\section{METHODS}

A Markov model was constructed to simulate costs and changes in BCVA over a 15-year period in a hypothetical cohort of patients with DME (figure 1), 


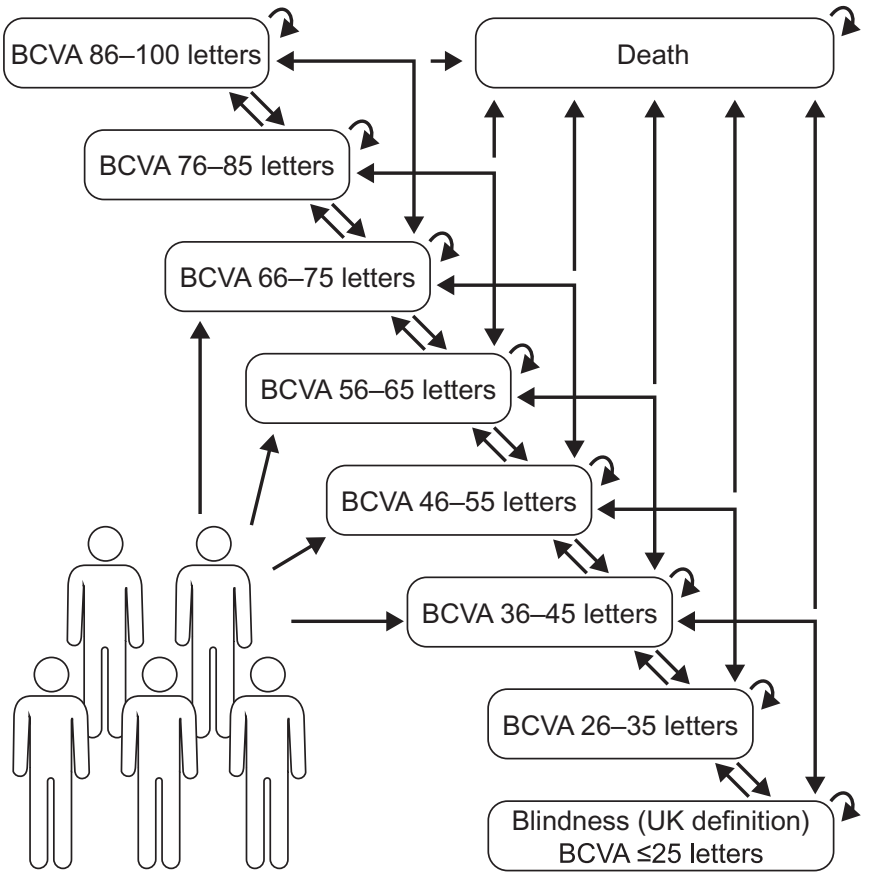

RESTORE patient

baseline characteristics

(BCVA 39-75 letters)

Figure 1 Markov model structure. Health states are defined by best corrected visual acuity (BCVA) in the treated eye. Patients enter the model at treatment start where they are assumed to have BCVA as in RESTORE ( $>39$ letters and $\leq 75$ letters). BCVA is evaluated at 3-monthly intervals. After each cycle, patients may transition to any other health state including death; the probability of moving from health state $A$ to health state $B$ is based on RESTORE data (baseline to month 12) and literature.

based on data from the RESTORE trial (in which $87.5 \%$ of patients had type 2 diabetes and average baseline glycated haemoglobin was $7.3 \%$ ). The average age at entry to the model was 63 years, consistent with patient characteristics in RESTORE. The model also assumed the same baseline BCVA distribution, but excluded patients with BCVA $>75$ letters, consistent with guidance from the ranibizumab summary of product characteristics that such patients may benefit less from treatment than those with baseline BCVA $\leq 75$ letters. ${ }^{6}$

The model framework allocated eight linear health states defined by BCVA in the treated eye using a set of 10-letter (twoline) categories (table 1). Movement of patients from one health state to another was determined by transition probabilities that depended on the effectiveness of treatment and natural BCVA changes over time. A 10-letter range was used to categorise health states based on evidence that a 10-letter BCVA score loss is associated with a substantial decline in health-related quality of life (eg, loss of functional ability, increasing dependency, role limitations and impaired mental health). ${ }^{15}$ Each health state was assigned a quality-of-life index and cost. Costs and outcomes were accrued over 3-month cycles, applying half-cycle corrections. The time horizon in the base case was 15 years; although a lifetime horizon could be justified given the chronic nature of the condition, we selected a more conservative approach for the base case because of the lack of evidence on long-term prognosis.

The key inputs and assumptions are summarised in table 2. Patients were assumed to receive ranibizumab treatment in year 1 at the frequency observed in RESTORE. In year 2, patients
Table 1 Utility by BCVA in treated eye

\begin{tabular}{|c|c|c|c|}
\hline $\begin{array}{l}\text { Health state defined } \\
\text { by BCVA category } \\
\text { (letters; treated eye) }\end{array}$ & $\begin{array}{l}\text { RESTORE* } \\
\text { Mean utility (SE) }\end{array}$ & Lloyd et al ${ }^{12} \dagger$ & Brown et $a l^{13} \S$ \\
\hline $1: 86-100$ & $0.860(0.034) \ddagger$ & 0.830 & 0.839 \\
\hline 2: $76-85$ & $0.860(0.014)$ & 0.750 & 0.839 \\
\hline 3: $66-75$ & $0.813(0.012)$ & 0.750 & 0.783 \\
\hline $4: 56-65$ & $0.802(0.014)$ & 0.715 & 0.783 \\
\hline $5: 46-55$ & $0.770(0.018)$ & 0.680 & 0.732 \\
\hline $6: 36-45$ & $0.760(0.027)$ & 0.680 & 0.681 \\
\hline $7: 26-35$ & $0.681(0.053)$ & 0.530 & 0.630 \\
\hline 8: $0-25$ & $0.547(0.083)$ & 0.340 & 0.579 \\
\hline
\end{tabular}

*Utility scores were calculated based on EQ-5D scores in RESTORE; EQ-5D scores were converted to utilities using social tariffs measured in a UK population. ${ }^{14}$ Mean utility for each BCVA state was calculated using a regression technique for repeated measurements at baseline, month 3, month 6 and month 12. Data from several measurement points were pooled to cover all possible health state transitions with a sufficient sample size. A possible trend effect in the pooled data was rejected $(p<0.05)$.

†Patients underwent a Snellen visual acuity (VA) assessment and were categorised based on the better-seeing eye. Some adjustments were made to published values in order to convert VA ranges in Lloyd et al (obtained in a population of patients with diabetic retinopathy) to health states as defined in the current model.

SUtilities were elicited from patients with diabetic retinopathy. Patients underwen a Snellen VA assessment and were categorised based on the better-seeing eye. Some adjustments were made to published values in order to convert VA ranges to health states as defined in the current model.

$\ddagger$ Restricted to being greater than or equal to the utility in health state 2 . BCVA, best corrected visual acuity.

were assumed to need fewer injections, as observed in the Diabetic Retinopathy Clinical Research Network (DRCR.net) protocol I study (which included patients with comparable baseline demographics to RESTORE). ${ }^{1}$ A proportionately smaller number of monitoring visits was therefore assumed in year 2. After year 2, laser therapy was assumed to be administered as required in all arms, with no further need for ranibizumab; the assumed number of monitoring visits was further reduced accordingly.

The average BCVA achieved in year 1 was assumed to be maintained during year 2, as was observed in the DRCR.net protocol I study. ${ }^{1}$ After year 2 , all arms of the model followed natural disease history based on 4-year health state transition outcomes modelled from the Wisconsin Epidemiologic Study of Diabetic Retinopathy (WESDR) reports. ${ }^{17-19}$ Transition probabilities were calibrated to adjust for the improvement in diabetes management since the WESDR reports (see Supplementary Methods), and predicted that around $30 \%$ of patients would be expected to exhibit a worsening in BCVA of at least 10 letters and $20 \%$ of patients would show an improvement of at least 10 letters over a 4-year time horizon (Supplementary table 1).

Treatment discontinuation rates observed in RESTORE were applied to the model in year 1; it was assumed there would be no additional withdrawals from treatment in year 2. Adverse events were assumed to have a negligible impact on the cost-effectiveness of ranibizumab therapy based on the established safety profile of ranibizumab in clinical trials in DME and in wet agerelated macular degeneration, an indication for which ranibizumab is also licensed. ${ }^{120-22}$

Mortality was estimated by adjusting general UK population death rates according to the increased $R R$ of death in patients with DME. Mulnier et al estimated an increased mortality (HR 1.93 ) in a UK type 2 diabetes population relative to patients without diabetes, ${ }^{23}$ while Hirai et al estimated an HR of 1.27 for death in patients with clinically significant macular oedema (CSME) and diabetes relative to diabetic patients without CSME. ${ }^{24}$ We calculated a $2.45 \mathrm{RR}$ of death in a DME population by multiplying these two ratios. 
Table 2 Key model inputs and assumptions

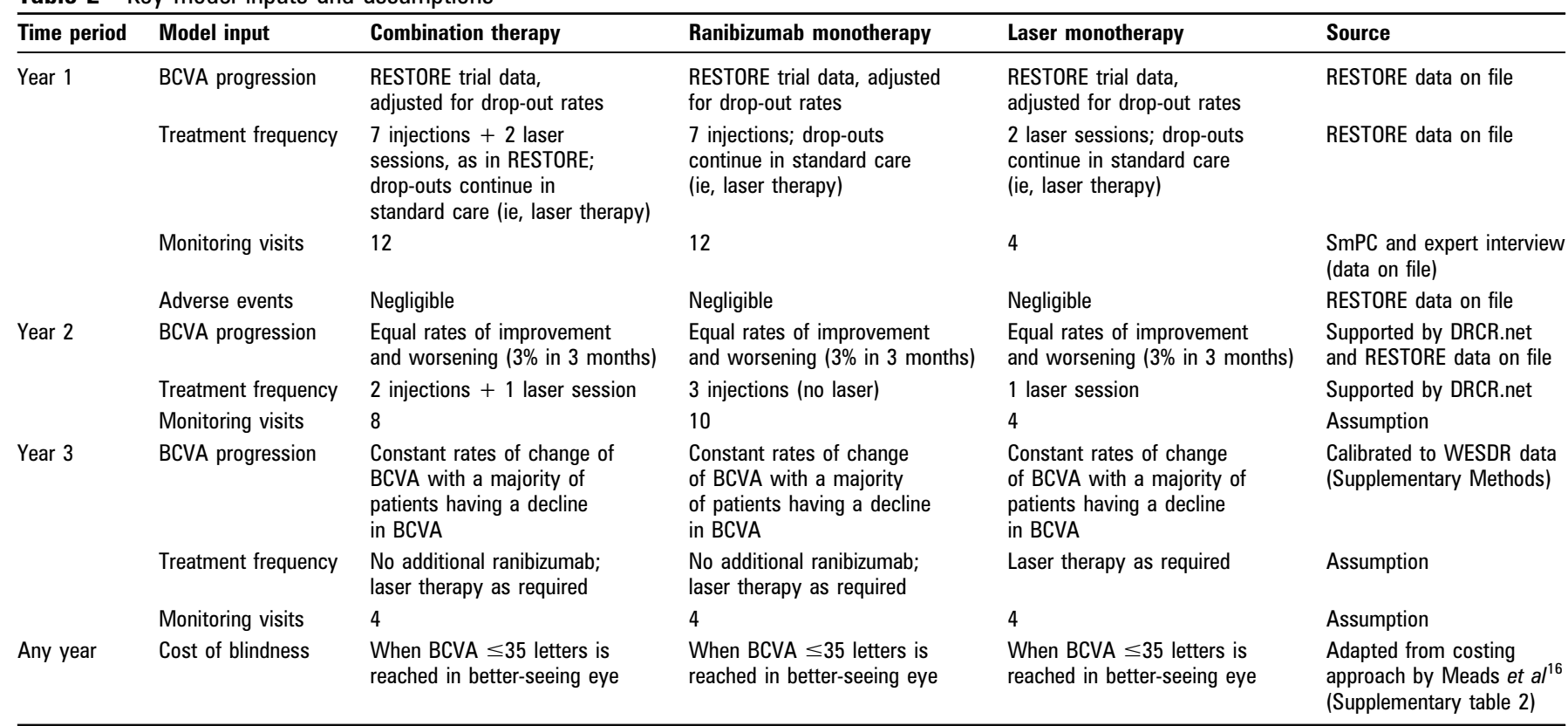

Patient-level changes in BCVA data in RESTORE were used to derive transition probabilities in year 1.

After year 1, long-term changes in BCVA were simulated assuming categorisation into one of three possible outcomes: $\geq 10$ letters improvement within 3 months (one health state up), $\geq 10$ letters worsening within 3 months (one health state down) or no change exceeding 10 letters within 3 months.

BCVA, best corrected visual acuity; DRCR, Diabetic Retinopathy Clinical Research Network; SmPC, summary of product characteristics; WESDR, Wisconsin Epidemiologic Study of Diabetic Retinopathy.

Utility scores were calculated based on patient-reported outcomes data from RESTORE (table 2), in which patients completed the EuroQoL (EQ-5D) questionnaire at baseline and months 3, 6 and 12. Individual EQ-5D health scores were converted into utility scores using preferences from a UK population survey; ${ }^{14}$ mean utility scores were calculated for each health state (table 1). As these states were defined by BCVA in the treated eye, of which $67.2 \%$ were the worse-seeing eye at baseline, this method established an association between utility and BCVA changes in the treated eye.

Health state costs included the costs of treatment and monitoring (Supplementary tables 3-6), and the costs associated with blindness (Supplementary table 2). Treatment costs included the costs of ranibizumab (Novartis UK, personal communication) and its administration, laser therapy and investigative procedures. Monitoring costs, including consultation and procedure costs, were estimated from the UK National Health Service Reference Costs. ${ }^{25}$ Costs of blindness included those incurred by the UK National Health Service for items such as low-vision aids, low-vision rehabilitation, residential or home care, depression and hip fracture/replacement as listed in the costing study by Meads and Hyde. ${ }^{16}$ Where older cost estimates were used, these were inflated to 2010 prices using the Hospital and Community Health Services index. ${ }^{26}$ The cost of blindness would be incurred only in patients reaching health states with BCVA $\leq 35$ letters (Snellen $\leq 6 / 60$ ) in the better-seeing eye. However, as the study assesses treatment response according to the enrolled eye, the proportion of patients reaching this level within the time horizon of the model is therefore uncertain. As such, the base case model adjusts for the cost of blindness on the basis of treated eyes reaching the BCVA $\leq 35$-letter threshold. As with other model parameters subject to uncertainty, deviations from this assumption were explored in sensitivity analyses (Supplementary table 7).

The main outcome measure was the incremental costeffectiveness ratio (ICER), expressed as the additional cost per quality-adjusted life-year (OALY) gained by one treatment over another. An annual 3.5\% discount rate was applied for future costs and utilities, consistent with the standard UK approach.

Univariate sensitivity analyses assessed the uncertainty around specific data sources by exploring the effects on the ICER of inputs, as shown in table 3. Probabilistic sensitivity analysis assessed the overall uncertainty about the ICER based on variations in individual input parameters; details of the applied distributions and results are provided in Supplementary table 7.

\section{RESULTS}

The model predicted that after 1 year, a greater proportion of patients treated with ranibizumab monotherapy or combination therapy would have BCVA >65 letters (Snellen score $>6 / 18$ ) compared with patients treated with laser monotherapy (48\% and $47 \%$, respectively, versus $38 \%$ in the laser arm) (figure 2). After 15 years, the proportion with severe visual impairment in the treated eye $(\mathrm{BCVA}<35$ letters, Snellen $<6 / 60)$ was predicted to be $12 \%$ and $13 \%$ in the ranibizumab monotherapy and combination therapy groups, respectively, versus 19\% in the laser group.

Ranibizumab monotherapy was associated with an incremental gain of 0.17 OALY and cost of $£ 4191$, corresponding to an ICER of $£ 24028$ per OALY gained relative to laser monotherapy (table 3). Combination therapy provided an incremental gain of 0.13 OALY over laser monotherapy for an incremental cost of $£ 4695$, leading to an ICER of $£ 36106$ per OALY gained.

Univariate sensitivity analyses showed that the model was stable and that ICERs were most sensitive to changes in the number of injections and time horizon (table 3). Using utilities elicited by Lloyd et $a l^{12}$ and Brown et al ${ }^{13}$ in patients with diabetic retinopathy led to greater OALY gains and lower ICERs for ranibizumab monotherapy or combination therapy relative to laser monotherapy. Probabilistic sensitivity analysis confirmed the model's robustness (Supplementary figure 1). Assuming a willingness-to-pay threshold of $£ 30000$ per OALY gained, the 
Table 3 Cost-effectiveness: base case and sensitivity analyses*

\begin{tabular}{|c|c|c|c|c|c|c|}
\hline Assumption/parameter & Base case & Sensitivity analyses & Incremental cost & Incremental OALY & ICER & ICER (\% change) \\
\hline \multicolumn{7}{|c|}{ Ranibizumab monotherapy versus laser monotherapy } \\
\hline Base case & - & - & $£ 4191$ & 0.17 & $£ 24028$ & - \\
\hline $\begin{array}{l}\text { Discount rate of future costs } \\
\text { and benefits }\end{array}$ & $3.50 \%$ & $0 \%-5 \%$ & $£ 3593-£ 4383$ & 0.16 to 0.16 & $£ 17051-£ 27042$ & $-29 \%$ to $+13 \%$ \\
\hline Time horizon & 15 years & $10-20$ years & $£ 4738-£ 3991$ & 0.14 to 0.19 & $£ 33139$ to $£ 21343$ & $+38 \%$ to $-11 \%$ \\
\hline Cost of blindness & $£ 6477$ & $-25 \%$ to $+25 \%$ & $£ 4868-£ 3515$ & 0.17 to 0.17 & $£ 27907-£ 20150$ & $+16 \%$ to $-16 \%$ \\
\hline Long-term progression of VA & Declining & Constant or increasing & $£ 4487-£ 4693$ & 0.17 to 0.17 & $£ 26198-£ 28413$ & $+9 \%$ to $+18 \%$ \\
\hline $\begin{array}{l}\text { Total number of ranibizumab } \\
\text { injections }\end{array}$ & 10 & -4 injections to +4 injections & $£ 2171-£ 6774$ & 0.17 to 0.17 & $£ 12446-38836$ & $-48 \%$ to $+62 \%$ \\
\hline Baseline age & 63 years & 58 years & $£ 3767$ & 0.20 & $£ 19259$ & $-20 \%$ \\
\hline \multirow[t]{2}{*}{ Source of utilities } & RESTORE & Lloyd et al ${ }^{12}$ & $£ 4191$ & 0.22 & $£ 19238$ & $-20 \%$ \\
\hline & RESTORE & Brown et al ${ }^{13}$ & $£ 4191$ & 0.19 & $£ 21953$ & $-9 \%$ \\
\hline \multicolumn{7}{|c|}{ Combination therapy versus laser monotherapy } \\
\hline Base case & - & - & $£ 4695$ & 0.13 & $£ 36106$ & - \\
\hline $\begin{array}{l}\text { Discounting of future costs } \\
\text { and benefits }\end{array}$ & $3.50 \%$ & $0 \%-5 \%$ & $£ 4271-£ 4828$ & 0.16 to 0.12 & $£ 26957-£ 40096$ & $-25 \%$ to $+11 \%$ \\
\hline Time horizon & 15 years & $10-20$ years & $£ 5133-£ 4507$ & 0.10 to 0.13 & $£ 49294-£ 34135$ & $+37 \%$ to $-5 \%$ \\
\hline Cost of blindness & $£ 6477$ & $-25 \%$ to $+25 \%$ & $£ 5050-4340$ & 0.13 to 0.13 & $£ 38833-£ 33378$ & $+8 \%$ to $-8 \%$ \\
\hline Long-term progression of VA & Declining & Constant or increasing & $£ 5091-£ 5276$ & 0.13 to 0.12 & $£ 40852-£ 44071$ & $+13 \%$ to $+22 \%$ \\
\hline $\begin{array}{l}\text { Total number of ranibizumab } \\
\text { injections }\end{array}$ & 9 & -4 injections to +4 injections & $£ 3165-£ 7260$ & 0.13 to 0.13 & $£ 24340-£ 55828$ & $-33 \%$ to $+55 \%$ \\
\hline Baseline age & 63 years & 58 years & $£ 4393$ & 0.15 & $£ 29952$ & $-17 \%$ \\
\hline \multirow[t]{2}{*}{ Source of utilities } & RESTORE & Lloyd et $a l^{12}$ & $£ 4695$ & 0.16 & $£ 28778$ & $-20 \%$ \\
\hline & RESTORE & Brown et al ${ }^{13}$ & $£ 4695$ & 0.16 & $£ 29576$ & $-18 \%$ \\
\hline
\end{tabular}

*Incremental cost measures the additional cost of ranibizumab monotherapy or combination therapy compared with laser monotherapy in the modelled time horizon (15 years in base case) Incremental QALY measures the corresponding QALY gain when ranibizumab monotherapy or combination therapy is compared with laser monotherapy. The ICER is calculated by dividing the incremental cost by the incremental QALY. The ICER is interpreted as the cost of achieving an additional year of life in perfect health.

ICER, incremental cost-effectiveness ratio; QALY, quality-adjusted life-year; VA, visual acuity.

probability that ranibizumab monotherapy would be costeffective relative to laser monotherapy was estimated to be $64 \%$ (Supplementary figure 2); the corresponding probability for combination therapy relative to laser monotherapy was $42 \%$.

\section{DISCUSSION}

This is the first study to evaluate the cost-effectiveness of an anti-VEGF agent for the treatment of patients with DME causing visual impairment. Our economic model, which was based on data from the RESTORE clinical trial, shows that ranibizumab monotherapy provides superior improvements in visual acuity and is cost-effective relative to the current standard of care, laser photocoagulation. Ranibizumab monotherapy was associated with an ICER of $£ 24028$ per OALY gained relative to laser therapy alone, a value within the willingness-to-pay threshold of $£ 30000$ per OALY gained that has generally been considered costeffective in the UK. The results of our model may be applicable to a broad spectrum of patients with visual impairment due to DME, as the RESTORE trial showed consistent efficacy of ranibizumab therapy across patient subgroups based on a range of demographic and disease characteristics.

The cost-effectiveness of combined ranibizumab and laser therapy was less favourable than that of ranibizumab monotherapy (ICER $£ 36106$ per OALY gained relative to laser therapy alone). While this in part reflects higher treatment costs, combination therapy also provided a lower predicted OALY gain than ranibizumab monotherapy ( 0.13 vs 0.17 OALY over the 15 year time horizon in the base case). A possible explanation for these results is the observation in RESTORE that combination therapy provided smaller improvements in BCVA in patients who had received prior laser therapy than those who had not (4.7 vs 6.9 letters gained, respectively). ${ }^{11}$ In RESTORE, the proportions of patients who received prior laser therapy were $52 \%$ (ranibizumab monotherapy), 47\% (combination therapy) and $43 \%$ (laser therapy).
Sensitivity analyses showed that the model results were robust to reasonable alterations in inputs and assumptions; ICERs were particularly sensitive to changes in the number of ranibizumab injections and the time horizon of the model. The base case assumed an average of 10 ranibizumab injections over 2 years, based on data from the DRCR.net protocol I study. ${ }^{1}$ Ranibizumab monotherapy remained cost-effective (ICER below $£ 30000$ per OALY gained) up to a total of 13 injections. Increasing the number of injections beyond 13 resulted in an ICER outside the generally accepted threshold, emphasising that additional injections beyond 2 years of treatment will be an important cost driver. However, the current model is conservative in that it includes incremental costs for additional injections beyond year 2, but assumes no incremental benefit. The possible need for re-treatment beyond 2 years remains speculative; forthcoming data from the 2- and 3-year RESTORE follow-up and the DRCR.net 5-year data will improve our understanding of the likely duration of treatment. Longer time horizons would be expected to lead to improved cost-effectiveness of ranibizumab, because the benefits of improved vision accrue over time whereas treatment costs are incurred immediately once treatment is initiated.

We have identified only one previously reported cost-effectiveness study of interventions for DME. Sharma et al modelled the cost-effectiveness of laser therapy alone for DME, ${ }^{27}$ comparing early and deferred laser treatment with no treatment based on 3-year outcomes from the Early Treatment Diabetic Retinopathy Study. ${ }^{28}$ The model included health states defined by BCVA and applied a 40-year time horizon based on a population with a mean age of 47 years. Over this time horizon, laser treatment was predicted to provide a gain of 0.236 OALY and was considered highly cost-effective for DME relative to no treatment. Our results cannot be compared directly with the Sharma et $a l^{27}$ study because RESTORE did not include a 'no treatment' arm; moreover, our model applied a 15-year time 

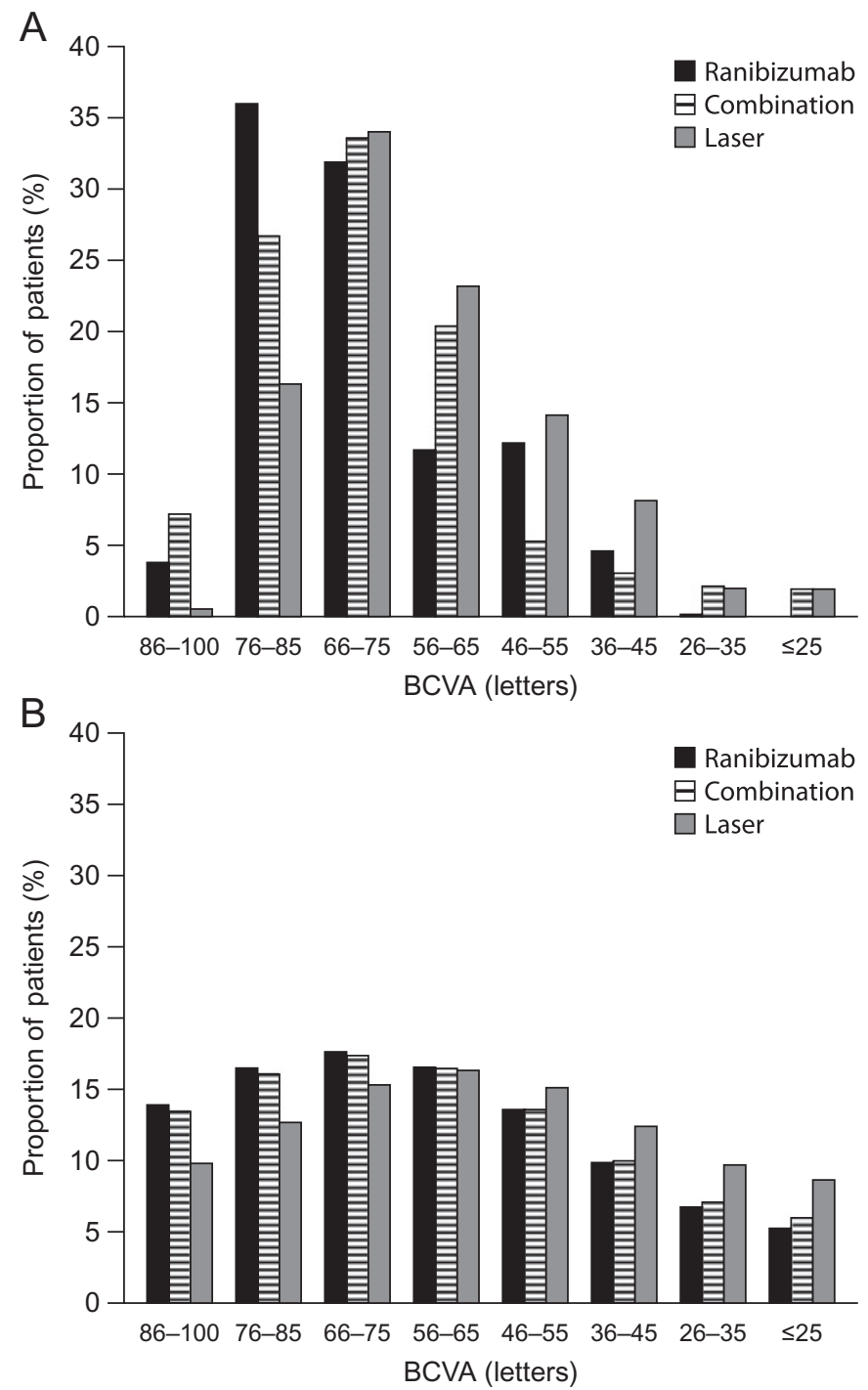

Figure 2 Modelled distribution by health states after (A) 1 and (B) 15 years. BCVA, best corrected visual acuity.

horizon from a baseline age of 63 years, consistent with RESTORE. However, if a baseline age of 47 years and time horizon of 40 years were applied to our model, as in the Sharma et $a^{27}$ model, the RESTORE model would predict a 0.26 OALY gain and an ICER of $£ 10412$ for ranibizumab monotherapy relative to laser therapy alone. This suggests that the costeffectiveness of ranibizumab would be higher in patients with DME who were younger than the average in RESTORE (63 years) because of their longer life expectancy.

Several methodological considerations should be noted. While treatment effectiveness was based on 1-year data from RESTORE, we projected long-term changes in BCVA using the best available clinical evidence. For year 2, the DRCR.net protocol I study supported the assumption that BCVA at 1 year was maintained on average to 2 years. ${ }^{1}$ BCVA changes in years 3-15 were based on reported progression in the WESDR study, which demonstrated a gradual decline in vision over time in DME. The rate of decline in BCVA was reduced by adjustment using data from both the Diabetes Control and Complications Trial and UK Prospective Diabetes Study to account for a less intensive systemic diabetes management regimen in the past relative to current practice. ${ }^{29-32}$ Sensitivity analysis showed that these assumptions had only a minor impact on the model results.

Utilities were estimated from the BCVA of treated eyes in RESTORE, irrespective of whether this was the better-seeing or worse-seeing eye. This approach links utility values directly to the eye receiving the intervention. The RESTORE study protocol required the worse-seeing eye to undergo intervention unless there was a medical contraindication; $32.8 \%$ of treated eyes were the better-seeing eye at baseline, and $37.3 \%$ were the better-seeing eye at 12 months. Using the better eye as reference is supported by quality of life studies in vision-related conditions, which have shown that the better eye is the major driver of overall quality of life and patient functioning. ${ }^{33}$ Indeed, patient-preference studies have shown that the utility gains associated with treating the worse-seeing eye are uncertain, even though good vision in two eyes confers better quality of life than good vision in only one eye. ${ }^{34}$ As expected, subdividing the elicited utilities in RESTORE by better-seeing or worse-seeing eye showed that patients being treated in the better-seeing eye reported lower utility at a given level of BCVA than those treated in the worse-seeing eye. Nevertheless, utility measured in patients treated in the worse-seeing eye demonstrated significant sensitivity to variation in the BCVA in the treated eye, comparable with that of patients treated in the better-seeing eye. Unfortunately, the small sample of RESTORE patients in each BCVA health state meant that the resulting utility functions were not sufficiently robust to allow separate cost-effectiveness analysis by better-seeing or worseseeing eye. It should also be stated that the option of not treating visual impairment in a better-seeing eye is not an ethical stance.

We could not find published utility estimates from specific populations with DME. We performed sensitivity analysis using utilities reported in two studies based on populations with diabetic retinopathy (which includes a wider population than DME). ${ }^{12}{ }^{13}$ Lloyd et al reported utilities elicited by the general population, while Brown et al reported utilities elicited by patients. This showed that applying the Lloyd et a ${ }^{12}$ utilities to the model greatly improved the cost-effectiveness of ranibizumab monotherapy, leading to a larger incremental gain of 0.22 OALY and a lower ICER of $£ 19238$ per OALY gained relative to laser therapy alone. Applying the Brown et al ${ }^{13}$ utilities also increased the OALY gain with ranibizumab and led to an ICER of $£ 21953$ per OALY gained for ranibizumab monotherapy versus laser therapy alone. The fact that the RESTORE utilities are less sensitive to BCVA decline may simply reflect the fact that a majority of the reference eyes in RESTORE were worseseeing, while all reference eyes in the Lloyd et $a l^{12}$ and Brown et $a l^{13}$ studies were better-seeing.

Limitations of this analysis should be considered. First, we modelled treatment of unilateral DME based on RESTORE data, but did not estimate the cost-effectiveness of treating bilateral DME. While $82.8 \%$ of patients in RESTORE had signs of bilateral DME at baseline, the proportion of patients who would have become eligible for treatment because of vision impairment in their fellow eye was not known. Treatment of both eyes may be relevant in many patients with bilateral DME, but there is a lack of evidence for the additional utility benefit of treating the fellow eye. Uncertainty also exists regarding the cost consequences of treating both eyes, where total cost is not likely to double given the possibility of achieving economies of scale from shared categorical spending, such as administrative and monitoring costs. An additional limitation was that the model assumed the cost of blindness would be incurred in patients 
reaching health states with BCVA $\leq 35$ letters (Snellen $\leq 6 / 60$ ) in the treated eye.

There is also uncertainty inherent in working with published results of studies, as was necessary for incorporating the DRCR Network Protocol I results, as opposed to using patient-level data. While analysis using patient level data is clearly preferable, incorporation of findings from studies in addition to RESTORE, such as the highly comparable DRCR study, provide very useful data for answering critical questions relating to clinical practice in a broad spectrum of patients with DME. It should be noted, however, that some subgroups, such as patients with the poorest glycaemic control (high glycated haemoglobin), were excluded from these trials.

In conclusion, the results of our economic model show that ranibizumab monotherapy is cost-effective relative to laser therapy alone in the treatment of DME causing visual impairment, while combined ranibizumab and laser therapy may be cost-effective depending on patient characteristics. The costeffectiveness of ranibizumab monotherapy or combination treatment is expected to be higher in younger patients who have a longer life expectancy. These findings have important practical implications, given the high socio-economic burden of DME and the need for new, cost-effective treatments that reduce long-term progression to blindness. Ongoing studies, such as the RESTORE extension, will provide additional clarification of current uncertainties such as the need for ranibizumab injections after 2 years and the likelihood of recurrent DME.

Acknowledgements The authors acknowledge the editorial support of Dr Richard White (Oxford PharmaGenesis Ltd) in collating the comments of the authors and finalising the manuscript for submission.

Contributors All authors contributed to the conception and design of the modelling, the analysis and interpretation of the clinical and economic data, participated in the development and writing of the manuscript, and approved the final draft for publication.

Funding This study was supported by Novartis Pharma AG, Basel, Switzerland.

Competing interests PM has received a consultancy fee from Novartis Pharma AG, Pfizer, Solvay and Allergan. He has also been paid lecture fees/honoraria by Novartis Pharma AG, Pfizer, Solvay and Allergan. LA has received unrestricted grants from Novartis Pharma AG. SKT is an employee of Novartis Pharmaceuticals Corporation, East Hanover, NJ, USA. KG is an employee of Novartis Pharmaceuticals, Frimley, UK. $\mathrm{RH}$ is an employee of Novartis Pharma AG, Basel, Switzerland and a Novartis shareholder. MK and HO were employed as consultants by Novartis Pharma AG for this study. MG was formerly employed by Novartis Pharma AG, Basel, Switzerland; current affiliation Amgen Inc., Thousand Oaks, CA, USA.

Provenance and peer review Not commissioned; externally peer reviewed.

\section{REFERENCES}

1. Elman MJ, Aiello LP, Beck RW, et al. Randomized trial evaluating ranibizumab plus prompt or deferred laser or triamcinolone plus prompt laser for diabetic macular edema. Ophthalmology 2010;117:1064-77.

2. Royal College of Ophthalmologists. Guidelines for Diabetic Retinopathy. London, UK: Royal College of Ophthalmologists, 2005.

3. Cheung N, Mitchell P, Wong TY. Diabetic retinopathy. Lancet 2010;376:124-36.

4. Minassian DC, Owens DR, Reidy A. Prevalence of diabetic macular oedema and related health and social care resource use in England. $\mathrm{Br} \mathrm{J}$ Ophthalmol. Published Online First: 20 May 2011. doi:10.1136/bjo.2011.204040

5. Lanzetta P, van Nuys K, Tran I, et al. The Economic Burden of Diabetic Macular Edema from a U.S. Private Payer Perspective. Fort Lauderdale, FL, USA: Association for Research in Vision and Ophthalmology (ARV0), Poster A370, 2011.

6. Novartis Pharma UK. Lucentis $10 \mathrm{mg} / \mathrm{mL}$ Solution for Injection-Summary of Product Characteristics. Frimley, UK: Novartis Pharma UK, 2011.
7. Aiello LP, Bursell SE, Clermont A, et al. Vascular endothelial growth factorinduced retinal permeability is mediated by protein kinase $C$ in vivo and suppressed by an orally effective beta-isoform-selective inhibitor. Diabetes 1997:46:1473-80.

8. Collins PD, Connolly DT, Williams TJ. Characterization of the increase in vascular permeability induced by vascular permeability factor in vivo. $\mathrm{Br} \mathrm{J}$ Pharmacol 1993;109:195-9.

9. Funatsu H, Yamashita $\mathrm{H}$, Noma $\mathrm{H}$, et al. Increased levels of vascular endothelial growth factor and interleukin-6 in the aqueous humor of diabetics with macular edema. Am J Ophthalmol 2002;133:70-7.

10. Murata T, Ishibashi T, Khalil A, et al. Vascular endothelial growth factor plays a role in hyperpermeability of diabetic retinal vessels. Ophthalmic Res 1995;27:48-52.

11. Mitchell P, Bandello F, Schmidt-Erfurth U, et al. The RESTORE study: ranibizumab monotherapy or combined with laser versus laser monotherapy for diabetic macular edema. Ophthalmology 2011;118:615-25

12. Lloyd A, Nafees B, Gavriel $\mathrm{S}$, et al. Health utility values associated with diabetic retinopathy. Diabet Med 2008:25:618-24.

13. Brown MM, Brown GC, Sharma S, et al. Utility values and diabetic retinopathy Am J Ophthalmol 1999;128:324-30.

14. Kind P, Hardman G, Macran S. UK Popul Norms for EQ-5D Discussion Paper 172. 1999.

15. Matza LS, Rousculp MD, Malley K, et al. The longitudinal link between visual acuity and health-related quality of life in patients with diabetic retinopathy. Health Qual Life Outcomes 2008;6:95.

16. Meads C, Hyde C. What is the cost of blindness? Br J Ophthalmol 2003;87:1201-4

17. Klein R, Klein BE, Moss SE, et al. The Wisconsin epidemiologic study of diabetic retinopathy. II. Prevalence and risk of diabetic retinopathy when age at diagnosis is less than 30 years. Arch Ophthalmol 1984;102:520-6.

18. Klein R, Klein BE, Moss SE, et al. The Wisconsin epidemiologic study of diabetic retinopathy. III. Prevalence and risk of diabetic retinopathy when age at diagnosis is 30 or more years. Arch Ophthalmol 1984;102:527-32.

19. Moss SE, Klein R, Klein BE. The incidence of vision loss in a diabetic population. Ophthalmology 1988;95:1340-8.

20. Brown DM, Michels M, Kaiser PK, et al. Ranibizumab versus verteporfin photodynamic therapy for neovascular age-related macular degeneration: two-year results of the ANCHOR study. Ophthalmology 2009;116:57-65.

21. Regillo CD, Brown DM, Abraham P, et al. Randomized, double-masked, shamcontrolled trial of ranibizumab for neovascular age-related macular degeneration: PIER Study year 1. Am J Ophthalmol 2008;145:239-48.

22. Rosenfeld PJ, Brown DM, Heier JS, et al. Ranibizumab for neovascular age-related macular degeneration. N Engl J Med 2006;355:1419-31.

23. Mulnier HE, Seaman HE, Raleigh VS, et al. Mortality in people with type 2 diabetes in the UK. Diabet Med 2006;23:516-21.

24. Hirai $\mathbf{F E}$, Knudtson $\mathrm{MD}$, Klein $\mathrm{BE}$, et al. Clinically significant macular edema and survival in type 1 and type 2 diabetes. Am J Ophthalmol 2008;145:700-6.

25. National Health Service. NHS Reference Costs 2008-09. London, UK: Department of Health, National Health Service, 2010.

26. Curtis L. Unit Costs of Health and Social Care. Canterbury, UK: Personal Social Services Research Unit, University of Kent, 2010.

27. Sharma S, Brown GC, Brown MM, et al. The cost-effectiveness of grid laser photocoagulation for the treatment of diabetic macular edema: results of a patientbased cost-utility analysis. Curr Opin Ophthalmol 2000;11:175-9.

28. Early Treatment Diabetic Retinopathy Study Research Group. Photocoagulation for diabetic macular edema. Early Treatment Diabetic Retinopathy Study report number 1. Arch Ophthalmol 1985;103:1796-806.

29. Clarke PM, Gray AM, Briggs A, et al. A model to estimate the lifetime health outcomes of patients with type 2 diabetes: the United Kingdom Prospective Diabetes Study (UKPDS) Outcomes Model (UKPDS no. 68). Diabetologia 2004:47:1747-59.

30. Diabetes Control and Complications Trial Research Group. Retinopathy and nephropathy in patients with type 1 diabetes four years after a trial of intensive therapy. N Engl J Med 2000;342:381-9.

31. Stratton IM, Adler Al, Neil HA, et al. Association of glycaemia with macrovascula and microvascular complications of type 2 diabetes (UKPDS 35): prospective observational study. BMJ 2000;321:405-12.

32. UK Prospective Diabetes Study Group. Intensive blood-glucose control with sulphonylureas or insulin compared with conventional treatment and risk of complications in patients with type 2 diabetes (UKPDS 33). Lancet 1998;352:837-53.

33. Bressler NM, Chang TS, Suner IJ, et al. Vision-related function after ranibizumab treatment by better- or worse-seeing eye: clinical trial results from MARINA and ANCHOR. Ophthalmology 2010;117:747-56 e4.

34. Brown MM, Brown GC, Sharma S, et al. Quality of life associated with unilateral and bilateral good vision. Ophthalmology 2001;108:643-7; discussion 647-8. 